\title{
Le cathéter migrateur
}

\section{The migratory catheter}

\author{
C. Rothmann - B. Weryha - O. Pierrard \\ Reçu le 5 août 2013 ; accepté le 31 octobre 2013 \\ (C) SFMU et Springer-Verlag France 2013
}

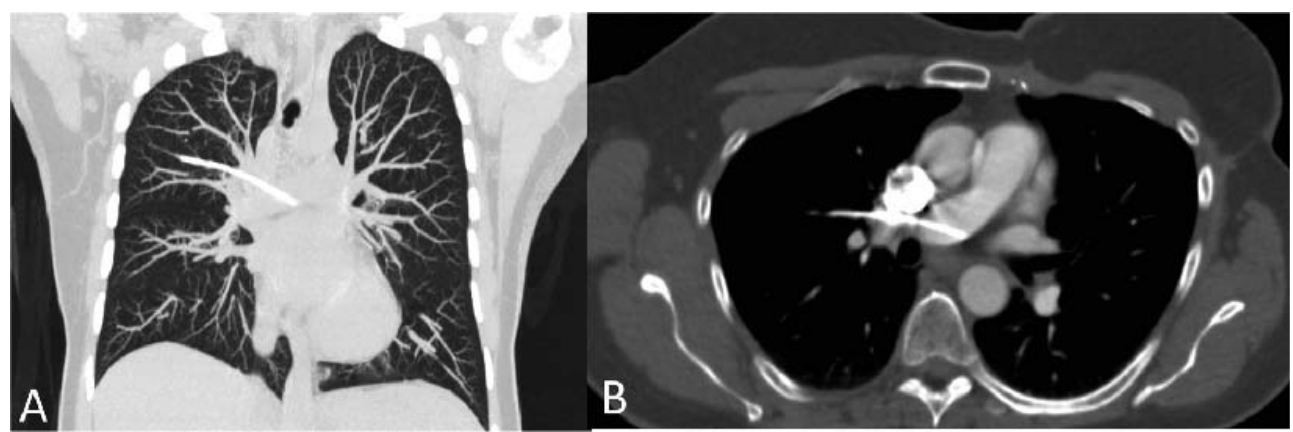

Fig. 1 Scanner thoracique en coupe frontale en fenêtre parenchymateuse (A) et en coupe axiale avec injection d'iode (B) : fragment de cathéter de $75 \mathrm{~mm}$ de long au niveau de la branche droite de l'artère pulmonaire et sortant de l'artère pour avoir un trajet intraparenchymateux longeant une bronche apicoventrale droite

Une patiente de 54 ans se présente aux urgences pour toux irritative et douleur thoracique, dans un contexte de carcinome canalaire infiltrant du sein gauche traité trois ans auparavant par chirurgie, radiothérapie et chimiothérapie à l'aide d'un cathéter port-a-cath. L'interrogatoire retrouve la notion de rupture du cathéter port- $a$-cath depuis au moins un mois (radiographie thoracique). L'examen cardiovasculaire et pulmonaire, l'ECG et la biologie sont sans anomalie. Le scanner thoracique sans et avec injection de produit de contraste iodé (Fig. 1) met en évidence une rupture avec migration d'un fragment distal de $75 \mathrm{~mm}$ de long du cathéter port-a-cath. La migration vasculaire des cathéters veineux centraux est une complication rare de leur utilisation, décrite depuis 1954 [1]. Elle succède à la rupture du cathéter, le plus souvent au niveau de l'espace entre la clavicule et la première côte (« syndrome du défilé »), au niveau du point d'entrée du cathéter dans la veine, où la friction mécanique contre le cathéter est la plus forte. Le déplacement extravasculaire de ce genre de dispositif est encore plus exceptionnel. Si le fragment migré du cathéter est intravasculaire, le traitement repose d'abord sur la radiologie interventionnelle endovasculaire. En cas de situation extravasculaire ou d'échec du traitement précédent, le traitement chirurgical doit être discuté [2].

\section{Références}

1. Turner DP, Sommers SC (1954) Accidental passage of polyethylene catheter from cubital vein to right atrium. N Engl J Med 251:744

2. Nayeemuddin M, Pherwani AD, Asquith JR (2013) Imaging and management of complications of central venous catheter. Clin Radiol 68:529-44 